Relations industrielles

Industrial Relations

\title{
La Ville : phénomène économique, par Jean Rémy, Éditions Vie Ouvrière, Bruxelles, 1966, 297 pages.
}

\section{Pierre Dionne}

Volume 22, numéro 2, 1967

URI : https://id.erudit.org/iderudit/027792ar

DOI : https://doi.org/10.7202/027792ar

Aller au sommaire du numéro

Éditeur(s)

Département des relations industrielles de l'Université Laval

ISSN

0034-379X (imprimé)

1703-8138 (numérique)

Découvrir la revue

Citer ce compte rendu

Dionne, P. (1967). Compte rendu de [La Ville : phénomène économique, par Jean Rémy, Éditions Vie Ouvrière, Bruxelles, 1966, 297 pages.] Relations industrielles Industrial Relations, 22(2), 301-302. https://doi.org/10.7202/027792ar

Tous droits réservés (C Département des relations industrielles de l'Université Laval, 1967
Ce document est protégé par la loi sur le droit d'auteur. L'utilisation des services d'Érudit (y compris la reproduction) est assujettie à sa politique d'utilisation que vous pouvez consulter en ligne.

https://apropos.erudit.org/fr/usagers/politique-dutilisation/ 
beaucoup d'attention. Néanmoins, tous ceux qui s'intéressent aux problèmes de l'automation y puiseront des données fondamentoles sur cette question en U.R.S.S.

\section{Pierre DIONNE}

Travail et automation - Etudes de cas sur l'évolution technique: tableaux analytiques, Bureau international du travail, cahier no 2 , Genève 1965, 89 pages.

L'une des première méthodes d'investigation utilisées aux fins de connaître les répercussions de l'automation, est l'étude de cas.

- Limitée ò une entreprise, ou encore à un groupe d'entreprises d'une même branche d'activité ou d'un même secteur, l'étude de cas a pour objet d'examiner en détail les conséquences de telle ou telle innovation technique dans l'entreprise, la branche d'activité ou le secteur considéré.

Le présent cahier présente sous forme de tableaux l'analyse de cent soixante études de cas, menée dons quatorze pays auprès d'entreprises de vingt-neuf branches d'activité

Du fait même de sa nature, l'analyse ne s'étend pas aux résultats auxquels ont pu conduire les études de cas considérées, pas plus qu'elle ne permet de tirer des conclusions. On peut penser toutefois que ce cahier, tout en fournissant aux chercheurs un instrument de référence, sera de quelque utilité pour l'établissement des programmes d'investigations futures.

\section{Pierre DIONNE}

\section{La participation de la collectivité à une} planification économique, Cahier de II.C.E.A., Montréal, no 3, février 1967, 144 pages.

Les pages de ce cahier ne contiennent pas des études sur la planification en tant que telle, mais bien un ensemble de réflexions sur la participation des collectivités à une pianification démocratique et efficace.

C'est à un effort de démystification que les outeurs tentent $d$ 'arriver par une approche concrète et pragmatique des problèmes. Le présent cahier ne s'adresse donc pas à des spécialistes de la planification, mais « au citoyen désireux d'être mieux informé et documenté sur la question, afin d'apporter ou meilleur de sa connaissance sa quote part de participation $\gg$.

$\mathrm{Ce}$ cahier se divise en deux parties. La première tente d'éclairer la notion même de planification démocratique, les modalités de consultation et la nature des orgonismes dont nous disposons au Canada et au Québec. La seconde partie décrit à partir des expériences du B.A.E.Q. et du Lac-St-Jean, le processus ou les méthodes utilisées, compte tenu de certains conditionnements et facteurs d'influence.

Bref, les textes que contient ce cahier, tendent à démontrer qu'aucun gouvernement ne peut se passer des corps intermédiaires dans l'édification de plans socio-économiques.

\section{Pierre DIONNE}

La Ville: phénomène économique, par Jean Rémy, Editions Vie Ouvrière, Bruxelles, 1966, 297 pages.

Cet ouvroge tente d'examiner la signification économique de la ville, surtout de la ville de grande dimension et d'en étudier le rôle accru tant pour le bien-être des populations que pour l'organisation efficace de lc production des divers biens.

Pour l'auteur le développement urbain, s'il s'opère adéquatement, doit valoriser l'urbanisation. La théorie de la croissance économique doit reconnaître le rôle des facteurs urbains et, la politique économique doit organiser la croissance urbaine de manière à réduire ou minimum les coûts qui en résultent.

Dans un premier temps, Jean Rémy analyse la signification de la juxtaposition spatiole - méthodes, étapes, causes - et le contenu des économies d'agglomération du point de vue de l'entrepreneur. Ensuite, il reprend les économies d'agglomération du point de vue du consommateur final.

L'auteur continue son étude en donnant le point de vue du sociologue sur la ville dans l'organisation et la croissance d'un ensemble social.

Dans un deuxième temps, l'analyse porte sur les diséconomies engendrées par l'agglomération et l'organisation de l'espace comme moyen d'y remédier.

Enfin, l'étude se termine en envisageant le développement urbain comme objectif d'une politique économique. 
Tout ou long de l'ourrage, on constote que sur chocun des aspects étudiés, le point de départ est une analyse inductive et le point d'orriver est l'apport théorique. C'est pourquoi, nous considérons ce volume comme un modèle de recherche sur ce sujet. II se révèlera très utile oux chercheurs d'autant plus qu'une excellente bibliographie est ò l'oppui de cette thèse.

\section{Pierre DIONNE}

Collective Bargaining Law in Conada, by A.W.R. Corrothers, Butterworths \& Company (Canada) Ltd., Toronto, 1965. 553 poges.

Ce volume du doyen Corrothers donne une description générale du droit du travail au Conada.

L'ouvrage est constitué de quatre pièces plus ou moins détachées: le développement historique du droit des rapports collectifs ou Conada - une étude des principales techniques juridiques propres au droit du trovail - une critique des principaux moyens d'expression de la grève (le piquet et le boycottage) - un aperçu sommaire du droit syndical. II faut signaler que cette étude tient compte du droit positif et de la jurisprudence des dix provinces et du Fédéral.

Dans sa partie historique, l'auteur met en relief les racines de notre droit du travail. A l'instar du droit anglais, c'est au Code criminel qu'il faut trouver le point de départ de la vie juridique du mouvement syndical. Après cette première phase, celle de la licéité de la coolition des travailleurs, on passe à la phase de l'organisation juridique des moyens de pression, puis à celle de l'institutionnalisotion des rapports collectifs en prenont cette fois les Etats-Unis comme modèle. En quelque soixante-dix pages, nous sommes mis en condition pour aborder la portie descriptive du droit octuel des ropports collectifs.

Cette deuxième partie est la plus importante puisqu'elle courre les $3 / 5$ du volume. L'auteur fait un exposé des principoles techniques juridiques et des instruments qui constituent l'infrastructure du droit des ropports collectifs.

On étudie à tour de rôle la Commission (noture et compétence), l'occréditotion, l'unité de négociotion, la négociation collective, la conciliation, la grève, la convention collective, la personnaité juridique du syn- dicat et le droit pénal du travail: Si l'outeur a voulu doner un simple expasé descriptif de ces différents sujets, il faut admettre qu'il a parfaitement réussi.

En trois cents pages, nous faisons le tour de toutes les provinces pour relever les points communs et les traits caractéristiques de chacune des institutions en cause. Cette étude comparative est faite en tenont compte à la fois du texte de loi, de lo doctrine et de la jurisprudence. En raison même de ce vaste domaine, il ne faut pas s'attendre à une analyse critique de chacune des institutions; on posse assez rapidement d'un sujet à l'outre. Cela suffit pour nous mettre en appétit et pour nous donner les principaux thèmes et les références nécessaires à une étude plus détaillée.

Ainsi, monsieur Carrothers consacre-t-il sept pages à la Loi des décrets de convention collective. Ce bref exposé suffit peutêtre à signaler la présence de cette loi à nos confrères des autres provinces, sans plus.

La troisième partie du volume constitue une étude plus opprofondie et mieux rodée du piquet et du boycottoge. Le titre de cette partie a The Limits of Labour Picketing and Boycotting "illustre bien la maîtrise de l'auteur sur ces questions. II s'agit de la reprise de problèmes plusieurs fois étudiés par monsieur Carrothers. Parce qu'il n'est pas obligé de faire le tour des dix provinces, l'auteur a pu approfondir davantage ce sujet.

La quatrième partie, la plus brève, est la plus nouvelle et la plus enrichissante. II s'agit de queques variations sur le thème des relations intersyndicales et des relations syndicat-syndiqués.

A mon avis, les chapitres 10 et 23 auraient pu être intégrés à cette dernière partie pour constituer une étude mieux structurée du droit syndical. Néanmoins, les grandes questions sont soulevées, à savoir: la nature des relations entre les centrales syndicales et I'association locale, puis l'outorité syndicale sur 'individu. Pourquoi et dans quelle mesure la volonté collective doit primer celle du solarié? Ces sujets constitueront l'objet de grandes études en droit du travail pour les prochaines années.

Est-ce utile d'ajouter que l'étudiant en droit ou en relations industrielles et le praticien doivent connaitre ce volume: la loi du moindre effort l'exige. 